\title{
Differential Regulation of Immune Signaling and Survival Response in Drosophila melanogaster Larvae upon Steinernema carpocapsae Nematode Infection
}

\author{
Shruti Yadav, Sonali Gupta and Ioannis Eleftherianos * (D) \\ Insect Infection and Immunity Lab., Department of Biological Sciences, The George Washington University, \\ Washington, DC 20052, USA; shrutiyadav@gwmail.gwu.edu (S.Y.); sonaligupta@gwmail.gwu.edu (S.G.) \\ * Correspondence: ioannise@gwu.edu; Tel.: +1-202-994-2367
}

Received: 9 December 2017; Accepted: 1 February 2018; Published: 8 February 2018

\begin{abstract}
Drosophila melanogaster is an excellent model to dissect the molecular components and pathways of the innate anti-pathogen immune response. The nematode parasite Steinernema carpocapsae and its mutualistic bacterium Xenorhabdus nematophila form a complex that is highly pathogenic to insects, including D. melanogaster. We have used symbiotic (carrying X. nematophila) and axenic (lacking X. nematophila) nematodes to probe the regulation of genes belonging to different immune signaling pathways in $D$. melanogaster larvae and assess the survival response of certain mutants to these pathogens. We found that both types of $S$. carpocapsae upregulate MyD88 (Toll), but not PGRP-LE (Imd); whereas axenic S. carpocapsae strongly upregulate Wengen (Jnk), Domeless (Jak/Stat), Dawdle (TGF $\beta$, Activin), and Decapentaplegic (TGF $\beta$, BMP). We further found that inactivation of Wengen and Decapentaplegic confers a survival advantage to larvae infected with axenic $S$. carpocapsae, whereas mutating PGRP-LE promotes the survival of larvae infected with symbiotic nematodes.
\end{abstract}

Keywords: Drosophila melanogaster; Steinernema carpocapsae; immune signaling; immune response

\section{Introduction}

Entomopathogenic nematodes are natural parasites that infect a range of insect species [1-3]. In recent years, they have also emerged as excellent models to dissect the molecular basis of nematode parasitism [4,5]. The entomopathogenic nematode Steinernema carpocapsae forms an excellent tool to study the molecular interplay between insect hosts and nematode parasites [6,7]. S. carpocapsae nematodes have developed mutualistic relationship with the Gram-negative bacteria Xenorhabdus nematophila, which live in the gut of the parasite and are potent pathogens of insects [6-9]. The nematodes infect insects at the infective juvenile stage, which is analogous to the Caenorhabditis dauer stage [10]. Once the nematode gains entry into an insect, it expels its associated bacteria in the hemolymph and both the nematode and bacteria multiply and secrete virulence factors that disarm the insect's immune system $[4,9,11]$. The bacteria also assist their nematode partner by producing molecules that break down insect tissues, thereby promoting feeding of the parasite and completion of its life cycle [12]. When the food source is depleted, the nematodes take up their mutualistic bacteria and exit the insect as infective juveniles in search of new susceptible hosts [10]. In addition, the development of tools to generate $S$. carpocapsae nematodes devoid of their mutualistic bacteria (axenic worms) has permitted elegant studies for the identification of host responses directed exclusively against the nematodes $[13,14]$.

Drosophila melanogaster is a well-established insect model to investigate the genetic basis of the innate immune response to a wide range of pathogens, including parasitic nematodes [15]. Although the molecular players involved in the recognition and defense against nematodes are 
not fully characterized, recent studies have identified genes/gene families in D. melanogaster larvae and adult flies that are induced in response to nematode infections [7,16,17]. In recent years, the use of S. carpocapsae as model of nematode parasitism has led to the identification of potential anti-nematode factors in insect hosts $[6,7,18]$. A recent transcriptomic study has identified several Drosophila larval genes that could be involved in the recognition of and defense against $S$. carpocapsae nematodes [7].

Here, we exposed $D$. melanogaster larvae to $S$. carpocapsae symbiotic or axenic nematodes and analyzed the transcriptional regulation of PGRP-LE (Immune deficiency pathway, Imd), MyD88 (Toll pathway), Wengen (Wgn, c-Jun N-terminal kinase signaling pathway; Jnk), Domeless (Dome, Janus kinase and signal transducer and activator of transcription pathway; Jak/Stat), Dawdle (Daw, Activin branch of the transforming growth factor beta pathway; TGF $\beta$ ) and Decapentaplegic (Dpp, Bone Morphogenetic Protein branch; TGF $\beta$ pathway), which belong to different immune signaling pathways. We also tested the survival ability of $D$. melanogaster loss-of-function mutant larvae against $S$. carpocapsae to assess the participation of certain signaling pathways in the D. melanogaster response to this parasitic nematode.

\section{Materials and Methods}

\subsection{Fly Strains}

Drosophila melanogaster strains used included Oregon, $w^{1118}, y w$, PGRP-LE (Bloomington Drosophila Stock Center; 33055; FBgn0030695), MyD88 ${ }^{c 3881}$ [19], Wgn (Vienna Drosophila Resource Center; v9152; FBgn0030941), UAS-Dome ${ }^{D N}$ [20], Daw (Kyoto Stock Center; 113490; FBgn0031461), and Dpp (Bloomington Drosophila Stock Centre; 397; FBgn0000490) [21] (Table A1). Wgn-RNAi and UAS-Dome ${ }^{D N}$ were crossed with ubiquitous Actin-Gal4 driver (y[1] w $\left.{ }^{*}\right] ; \mathrm{P}\{\mathrm{w}[+\mathrm{mC}]=$ Act5C-GAL4 $\} 25 \mathrm{FO} 1 / \mathrm{CyO}$, y[+]) [22]. Fly strains were grown on Drosophila media (Meidi LaboratoriesR Potomac, MD, USA) and approximately 10 granules of dry baker's yeast. All fly stocks were maintained at $25^{\circ} \mathrm{C}$ with a $12: 12-\mathrm{h}$ light:dark cycle. Late second-early third instar larvae were used for all experiments.

\subsection{Nematodes}

The entomopathogenic nematodes Steinernema carpocapsae carrying the mutualistic bacteria Xenorhabdus nematophila (symbiotic worms) were reared in the larvae of the greater wax moth Galleria mellonella [23]. Axenic S. carpocapsae nematodes (free of X. nematophila bacteria) were cultured using a previously described protocol [14]. These nematodes were washed with $1 \%$ bleach, and then rinsed five times with water to remove any traces of bacteria or bleach. Infective juveniles between 2 and 3 weeks of age were used for all infection experiments.

\subsection{Gene Transcript Level Analysis}

Reads Per Kilobase Million (RPKM) values for PGRP-LE, Myd88, Wgn, Dome, Daw, and Dpp genes were obtained from a recent RNA sequencing study [7], which involved the transcriptomic analysis of $D$. melanogaster larvae during the course of infection with $100 \mathrm{~S}$. carpocapsae symbiotic or axenic nematodes. The RPKM values for genes from larvae that were infected with either symbiotic or axenic nematodes were calculated relative to the RPKM values of the uninfected controls, which were set to 1.

\subsection{Survival Experiments}

Microtiter 96-well plates (Corning, Corning, NY, USA) were prepared by adding $100 \mu \mathrm{L}$ of $1.25 \%$ agarose to each well. Ten $S$. carpocapsae symbiotic or axenic nematodes suspended in $10 \mu \mathrm{L}$ of distilled water were added to each well containing a single larva. Addition of $10 \mu \mathrm{L}$ of distilled water served as negative control. The plate was covered with a Masterclear real-time PCR film (Eppendorf, Hamburg, Germany) and holes were poked for ventilation. Each experiment contained 10 larvae per immune mutant or background strain, per treatment. Survival experiments were monitored every $8 \mathrm{~h}$ for up to $64 \mathrm{~h}$ post-infection. Experiments were repeated at least three times. 


\subsection{Statistical Analysis}

Statistical tests for gene transcript level analysis were performed using one-way analysis of variance (ANOVA) and a Tukey post-hoc test for multiple comparisons. Statistics for survival experiments were carried out using log-rank (Mantel-Cox) and Chi-square tests. All statistical tests were conducted using the GraphPad Prism 7 software (GraphPad Software, La Jolla, CA, USA).

\section{Results}

3.1. Infection with S. carpocapsae Axenic Nematodes Results in Elevated Transcript Levels of Immune-Related Signaling Pathway Genes in D. melanogaster Larvae

We plotted the RPKM values for PGRP-LE (Imd), MyD88 (Toll), Wgn (Jnk), Dome (Jak/Stat), Daw (TGF $\beta$, Activin), and Dpp (TGF $\beta$, BMP) from D. melanogaster Oregon larvae infected for 6 or $24 \mathrm{~h}$ with either symbiotic or axenic $S$. carpocapsae. At $6 \mathrm{~h}$ post-infection, we found no difference in the expression of any of these genes in larvae infected with symbiotic or axenic nematodes compared to uninfected controls (Figure 1). At $24 \mathrm{~h}$ post-infection, the transcript levels of $P G R P-L E$ were slightly increased in larvae infected with symbiotic or axenic nematodes compared to the control larvae, although this induction was not statistically significant ( $p=0.2984$ and $p=0.1554$, respectively; Figure 1A). However, at $24 \mathrm{~h}$ post-infection the transcript levels of MyD88 were higher in larvae challenged with symbiotic $(p=0.0087)$ or axenic $(p=0.003)$ nematodes compared to controls (Figure 1B). Also, the transcript levels of $M y D 88$ were significantly increased in axenic $S$. carpocapsae infected larvae at $24 \mathrm{~h}$ compared to the $6 \mathrm{~h}$ time-point ( $p=0.008$; Figure $1 \mathrm{~B}$ ). Transcript levels of $W g n$ were significantly increased at $24 \mathrm{~h}$ post-axenic nematode infection compared to uninfected larvae ( $p=0.0165$; Figure 1C). Upon symbiotic nematode infection, Wgn was slightly upregulated compared to controls, but this increase was not statistically significant ( $p=0.1474$; Figure 1C). Dome and Daw were significantly upregulated at $24 \mathrm{~h}$ post-axenic nematode infection compared to control larvae ( $p=0.0108$; Figure 1D, $p=0.0068$; Figure 1E, respectively). Dome, Daw, and Dpp were upregulated in larvae infected with axenic nematodes at $24 \mathrm{~h}$ compared to $6 \mathrm{~h}(p=0.0474$; Figure 1D, $p=0.0353$; Figure 1E, $p=0.0372$; Figure $1 \mathrm{~F}$ ). We then validated the induction of all immune-related genes using quantitative RT-PCR and gene-specific primers (Figure A1). Consistent with the RNA-seq data, we did not find any differences in PGRP-LE transcript levels between larvae infected with symbiotic or axenic nematodes (Figure A1). In contrast to the RNA-seq, we did not find any differences in MyD88, Wgn, Daw, or Dpp transcript levels between uninfected control larvae and those infected with symbiotic nematodes. However, we found significant differences in Dome transcript levels in uninfected controls and larvae infected with symbiotic nematodes ( $p=0.0096$; Figure A1). Interestingly, qRT-PCR data showed a significant increase in the transcript levels of Dome compared to $\operatorname{Wgn}(p=0.0193)$ and $\operatorname{Dpp}(p=0.0014)$ in larvae infected with symbiotic nematodes (Figure A1). In larvae infected with axenic nematodes, Dome upregulation was significantly higher than $D p p(p=0.0324$; Figure A1). These results indicate that infection of $D$. melanogaster larvae with S. carpocapsae symbiotic or axenic nematodes results in upregulation of MyD88, Wgn, Dome, Daw, and Dpp (but not PGRP-LE), which are key components of signaling pathways that participate in innate immune processes. 
A

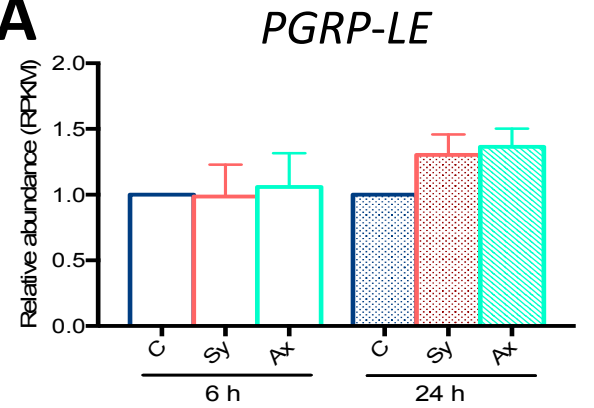

C

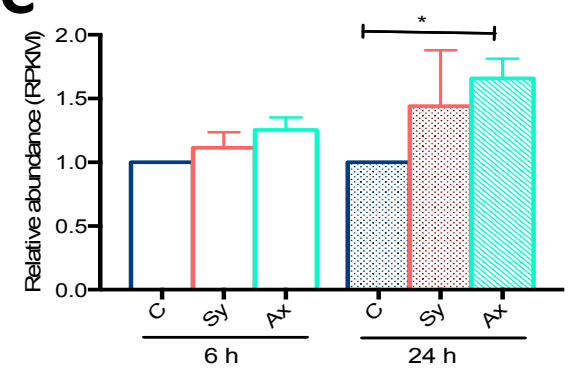

E

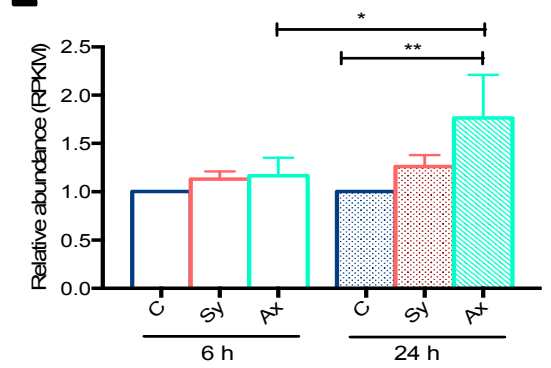

B

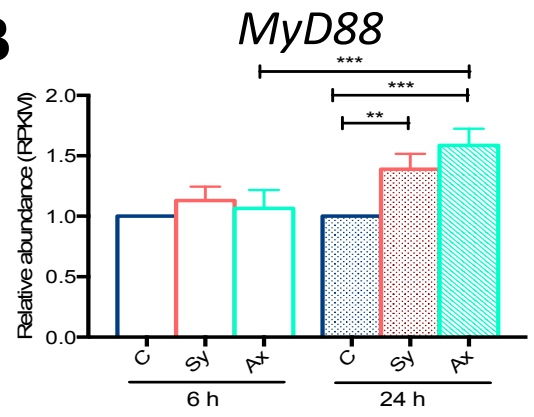

D

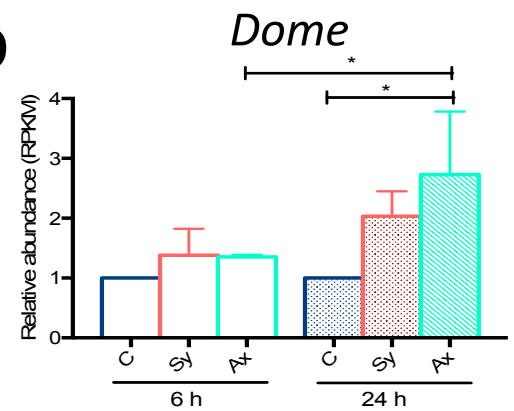

F

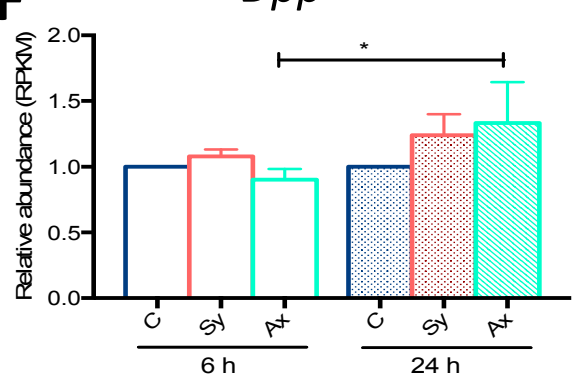

Figure 1. Relative gene transcript levels obtained using RNA-sequencing analyses on Drosophila melanogaster larvae infected with Steinernema carpocapsae symbiotic or axenic nematodes [7]. Relative transcript (Reads Per Kilobase Million; RPKM) levels of (A) PGRP-LE; (B) MyD88; (C) Wengen (Wgn); (D) Domeless (Dome); (E) Dawdle (Daw); and (F) Decapentaplegic (Dpp) were estimated in D. melanogaster larvae (Oregon strain) infected with 100 symbiotic (Sy) or axenic (Ax) S. carpocapsae nematodes at 6 and $24 \mathrm{~h}$ post-infection. Treatment with water served as control (C). Relative abundance was estimated as a ratio compared to the uninfected control larvae at 6 and $24 \mathrm{~h}$ post-treatment. Values represent means from three biological replicates and error bars represent standard deviations. Data were analyzed using one-way analysis of variance (ANOVA) and a Tukey post-hoc test for multiple comparisons using GraphPad Prism 7 software. ${ }^{*} p<0.05,{ }^{* *} p<0.01,{ }^{* * *} p<0.001$. Non-significant differences $(p>0.05)$ are not shown.

\subsection{D. melanogaster Imd, Jnk, and TGF $\beta$ Pathway Mutants Exhibit Enhanced Survival to Infection by} S. carpocapsae Nematodes

We infected D. melanogaster PGRP-LE, Myd88, Wgn, Dome, Daw, and Dpp loss-of-function mutant larvae and individuals from their respective background strains with $S$. carpocapsae symbiotic or axenic nematodes and monitored their survival response every $8 \mathrm{~h}$ for three days. Upon symbiotic nematode infection, the survival rate for PGRP-LE mutant larvae was significantly higher compared to their controls ( $p=0.0005$; Figure 2A). However, no significant differences in survival between PGRP-LE mutants and their controls were observed upon axenic nematode infection $(p>0.05$; Figure 2A). 
In contrast, there was no significant difference between the survival ability of $M y D 88$ mutant larvae and their background controls following infection with either symbiotic or axenic $S$. carpocapsae nematodes ( $p=0.607$ and $p=0.0804$, respectively; Figure 2B). After we confirmed significant reduction in $W g n$ transcript levels in larval progeny of Wgn-RNAi flies crossed with Actin-Gal4 compared to larval progeny of $w^{1118}$ flies crossed with this driver line (Figure A2), we found significantly higher survival rates for larvae expressing the $\mathrm{Wg} n$-RNAi construct compared to their controls following infection with axenic nematodes $(p=0.005$; Figure $1 C)$. We further observed no survival differences between Dome or Daw mutants and their controls in response to symbiotic or axenic nematodes ( $p>0.05$; Figure 2D,E). Although the Dpp mutant larvae and their controls succumbed similarly to infection by symbiotic nematodes, these mutants survived better the infection with axenic nematodes than their control individuals $(p<0.0291$; Figure 1F). These results indicate that inactivation of immune-related genes regulated by Imd, Jnk, and TGF $\beta$ pathways promotes the survival ability of D. melanogaster larvae to infection by $S$. carpocapsae nematodes.

A

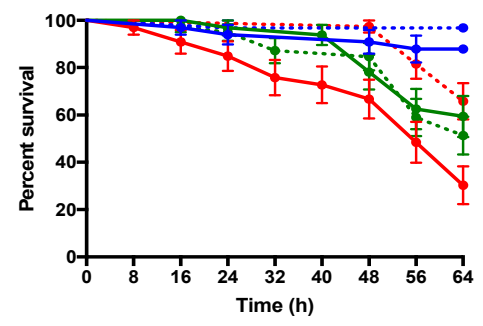

C

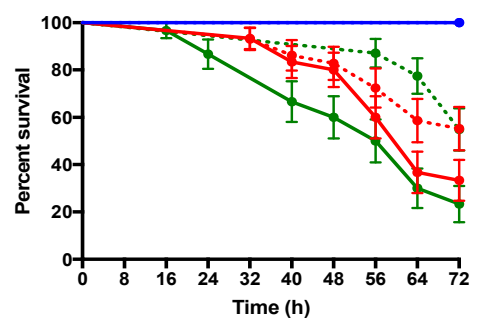

E

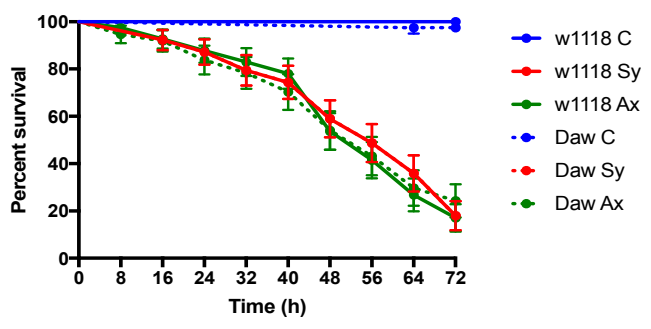

B

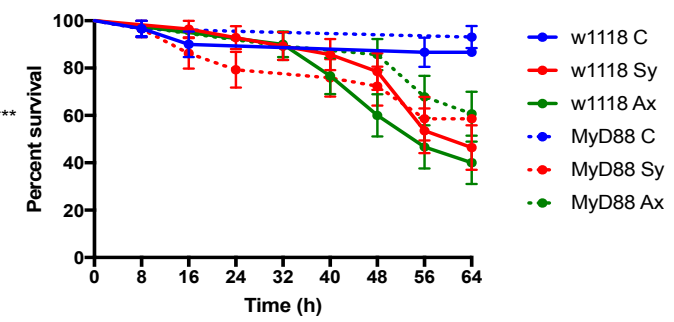

D

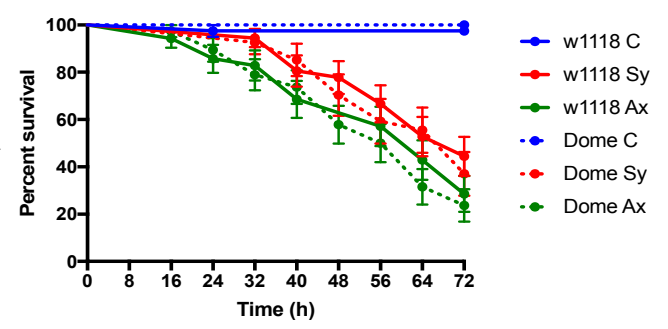

F

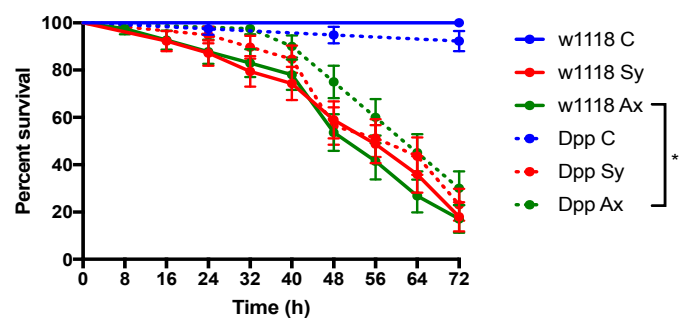

Figure 2. Survival response of Drosophila melanogaster immune mutant larvae infected with Steinernema carpocapsae symbiotic or axenic nematodes. Survival of late second to early third instar D. melanogaster larvae mutant for (A) PGRP-LE; (B) MyD88; (C) Wengen (Wgn); (D) Domeless (Dome); (E) Dawdle (Daw); or (F) Decapentaplegic (Dpp) genes, infected with symbiotic (Sy) or axenic (Ax) S. carpocapsae nematodes. Wgn-RNAi and UAS-Dome ${ }^{D N}$ were crossed with ubiquitous Actin-Gal4 driver. Treatment with water served as control (C). Larval survival was monitored every $8 \mathrm{~h}$ for up to $64 \mathrm{~h}$ post-infection. Values represent percent survival of infected larvae and data were analyzed using the Log-Rank test using GraphPad Prism 7 software. The means from three independent experiments are shown and bars represent standard errors. ${ }^{*} p<0.05,{ }^{* *} p<0.01,{ }^{* * *} p<0.001$. Non-significant differences $(p>0.05)$ are not shown. 


\section{Discussion}

A recent study investigating the immune response of $D$. melanogaster larvae to entomopathogenic nematodes focused on the induction of antimicrobial peptides (AMPs) as read-outs of the fly humoral immune response [6]. Here, we show that infection of D. melanogaster larvae with S. carpocapsae nematodes leads to the transcriptional induction of certain genes regulated by the Toll, Jnk, Jak/Stat, and TGF $\beta$ immune signaling pathways, but not of the Imd pathway. We also show that inactivation of $P G R P-L E$, which plays a key role in innate immunity by activating the Imd pathway, promotes the survival of $D$. melanogaster against symbiotic $S$. carpocapsae, whereas inactivation of Wgn (Jnk pathway) or $D p p$ (TGF $\beta$ pathway) genes promotes larval survival in response to axenic nematode infection.

The transcriptional regulation of immune genes in insects provides an indication for the potential involvement of specific immune signaling pathways in modulating the immune response against microbial invaders. Here, we used RNA-seq to determine the transcriptional induction of genes encoding receptors or signaling components upstream of the receptors in Imd, Toll, Jnk, Jak/Stat, and TGF $\beta$ pathways in D. melanogaster wild-type larvae infected with either $S$. carpocapsae symbiotic or axenic nematodes. Because RNA-seq is designed to estimate differential expression of a large number of genes, qRT-PCR can be used to validate the expression data [24]. Results from both RNA-seq and qRT-PCR analyses show a similar trend of upregulation for all genes tested. Using RNA-seq, we consistently found upregulation of Myd88, Wgn, Dome, Daw, and Dpp genes in larvae responding to axenic nematodes compared to symbiotic nematode infections. These results suggest that certain parasitic nematodes can broadly activate immune signaling in D. melanogaster larvae even in the absence of their mutualistic bacteria. Interestingly, S. carpocapsae nematodes carrying their mutualistic bacteria were recently shown to upregulate antimicrobial peptide coding genes in D. melanogaster larvae [6]. Our findings are in agreement with a previous study on D. melanogaster adult flies showing upregulation of certain antimicrobial peptide genes in response to Heterorhabditis bacteriophora axenic nematodes [25]. Although these immune signaling pathways are activated by both S. carpocapsae and H. bacteriophora, our current results indicate that PGRP-LE, a receptor of the Imd pathway, is not induced in response to $S$. carpocapsae infection. Thus, we speculate that $S$. carpocapsae nematodes can be detected by an unknown receptor in the Imd pathway, which leads to the induction of AMPs, as previously shown [6,7]. Further studies on the molecules involved in the recognition of entomopathogenic nematodes by the $D$. melanogaster immune system will be a future focus of our research.

The lower immune gene expression in response to symbiotic $S$. carpocapsae in the current study suggests that the mutualistic $X$. nematophila bacteria can interfere with the activation of immune signaling. Indeed, previous studies have shown that X. nematophila secretes a variety of toxins and virulence factors, many of which act on the insect immune system, thereby suppressing host immune genes that regulate important functions for fighting off the infection [26,27]. For example, the suppression of the Spodoptera exigua antimicrobial peptide genes by X. nematophila has been attributed to the activity of the metabolite benzylideneacetone, which is produced by the pathogen and acts through the eicosanoid pathway [26]. Also, suppression of cecropin expression in Manduca sexta is due to the presence of lrp, a global transcription factor in X. nematophila [27]. Interestingly, we have found that of the six immune genes tested, only PGRP-LE failed to show significant changes in transcript levels in response to $S$. carpocapsae infection. A previous study has demonstrated that the antimicrobial peptide gene diptericin is upregulated in D. melanogaster wild-type flies in response to X. nematophila infection and inactivation of diptericin confers high sensitivity to the mutant flies, suggesting that the Imd pathway is not only activated in response to X. nematophila, but it can also regulate the fly survival response to this pathogen [28]. The induction of immune genes in Toll, Jnk, Jak/Stat, and TGF $\beta$ signaling in our experiments suggests that these pathways could potentially be involved in the D. melanogaster response to $S$. carpocapsae nematode infection.

We further tested whether inactivating any of these genes affects the survival ability of $D$. melanogaster larvae to the nematode parasites. We found delayed mortality of PGRP-LE mutants 
compared to their background controls upon infection with symbiotic, but not axenic, S. carpocapsae nematode infection. These results indicate that upon inactivation of Imd signaling, the presence of $X$. nematophila bacteria in S. carpocapsae nematodes confers a pathogenicity disadvantage towards D. melanogaster. Previously, inactivation of either Toll or Imd pathways had no effect on the survival of D. melanogaster larvae upon infection with symbiotic H. bacteriophora [29]. These findings in combination with our current results suggest that the two entomopathogenic nematodes, H. bacteriophora and S. carpocapsae, elicit different responses in D. melanogaster, and that Toll and Imd signaling might participate in modulating distinct aspects of the insect anti-nematode immune response depending on the nematode species encountered. The conflicting results showing lack of PGRP-LE transcriptional induction by $S$. carpocapsae and the survival advantage of PGRP-LE deficient mutant larvae to $S$. carpocapsae infection suggests the existence of another Imd component that participates in the interaction with these parasitic nematodes.

In addition, infection with symbiotic $H$. bacteriophora elicits the transcriptional activation of distinct antimicrobial peptide genes in D. melanogaster larvae and adults. For example, metchnikowin was induced in D. melanogaster larvae in response to symbiotic $H$. bacteriophora, but very low to no induction was observed in adult flies [25,29]. Also, our finding that Dpp mutants have increased survival upon infection with axenic $S$. carpocapsae is in disagreement with our recent results showing that inactivation of $D p p$ decreases the survival ability of the mutant flies in response to H. bacteriophora axenic nematodes [30]. Thus, the developmental stage of the insect host can also regulate the types and magnitude of immune gene activation and response triggered against entomopathogenic nematode infection.

Interestingly, we find that inactivation of $W g n$ encoding the sole tumor necrosis factor receptor in $D$. melanogaster promotes the survival of larvae in response to infection by axenic S. carpocapsae, suggesting that Jnk signaling, which is involved in several D. melanogaster processes such as stress response, morphogenesis, and wound healing [31-33], might also interfere with the D. melanogaster response to certain nematode parasites. The induction of the Jnk-regulated pathway gene, puckered, in D. melanogaster adult flies in response to axenic $H$. bacteriophora reinforces the notion that Jnk signaling, through an unknown mechanism, might participate in the interaction with entomopathogenic nematodes [25].

Future work will focus on understanding the connection between immune pathway activation in D. melanogaster and regulation of the systemic immune function against entomopathogenic nematodes. It will be of particular interest to identify those genes that serve to recognize molecular patterns of entomopathogenic nematodes. The identification and characterization of the immune signaling molecular components that participate in the D. melanogaster defense against potent parasitic nematodes will potentially reveal novel anti-nematode mechanisms in other insects and perhaps even mammals.

\section{Conclusions}

In this study, we examined the regulation of PGRP-LE (Imd pathway), MyD88 (Toll pathway), Wgn (Jnk pathway), Dome (Jak/Stat pathway), Daw (Activin, TGF $\beta$ pathway), and Dpp (BMP, TGF $\beta$ pathway) genes in $D$. melanogaster larvae responding to infection with $S$. carpocapsae symbiotic or axenic nematodes. We also measured the survival response of mutant larvae against the parasitic nematodes. We have found upregulation of MyD88, Wgn, Dome, Daw, and Dpp, but not PGRP-LE, in $D$. melanogaster infected with the nematode parasites. We have also shown that inactivation of PGRP-LE, Wgn, and Dpp prolongs the survival of nematode-infected larvae. This work demonstrates the modulation of the D. melanogaster immune signaling pathways during infection with $S$. carpocapsae entomopathogenic nematodes. 
Acknowledgments: Research in the Eleftherianos Lab is funded by NIH and GWU grants. We thank the Vienna Drosophila RNAi Center, the Bloomington Drosophila Stock Center, the Kyoto Stock Center, and Dan Hultmark for kindly providing the $U A S-D o m e^{D N}$ strain. FlyBase is funded by the National Human Genome Research Institute at the U.S. National Institutes of Health (\#U41HG000739, PI W.M. Gelbart).

Author Contributions: S.Y. and I.E. conceived and designed the experiments; S.Y. and S.G. performed the experiments; S.Y. and S.G. analyzed the data; S.Y. and I.E. wrote the paper.

Conflicts of Interest: The authors declare no conflict of interest.

\section{Appendix A}

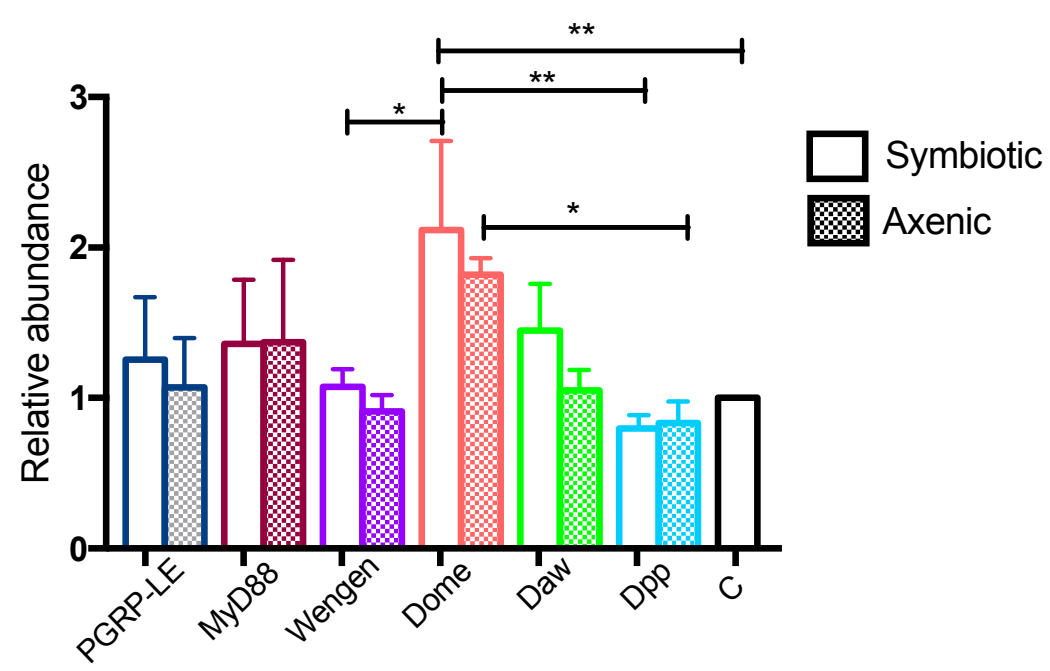

Figure A1. Validation of RNA-sequencing gene transcript levels in Drosophila melanogaster larvae infected with Steinernema carpocapsae symbiotic or axenic nematodes using qRT-PCR. Relative transcript levels of PGRP-LE, MyD88, Wengen, Domeless, Dawdle, and Decapentaplegic genes in D. melanogaster larvae infected with symbiotic or axenic $S$. carpocapsae nematodes at $24 \mathrm{~h}$ post-infection. Transcript levels are shown as relative abundance normalized to the housekeeping $R p L 32$ gene and expressed as a ratio compared to the uninfected control larvae for each strain. Values of uninfected control (C) samples are set to one. Values represent means from four biological replicates and error bars represent standard deviations. Data were analyzed using one-way analysis of variance (ANOVA) and a Tukey post-hoc test for multiple comparisons using GraphPad Prism 7 software. ${ }^{*} p<0.05,{ }^{* *} p<0.01,{ }^{* * *} p<0.001$. Non-significant differences $(p>0.05)$ are not shown.

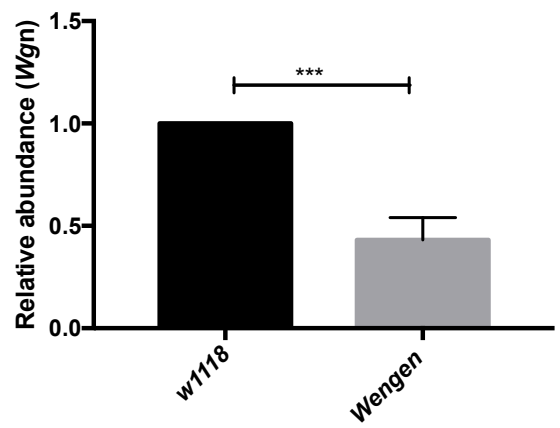

Figure A2. Validation of Wengen-RNAi line. Relative transcript levels of Wengen (Wgn) were measured in larval progeny of $w^{1118}$ and Wgn-RNAi flies crossed with Actin-Gal4 individuals. Transcript levels are represented relative to housekeeping gene $R p L 32$, and as a ratio compared to the progeny from the control cross. Values represent means from three replicates and error bar represents standard deviation. Data analysis was performed using unpaired $t$-test using GraphPad Prism 7 software. ${ }^{* *} p<0.001$. 


\section{Appendix B}

\section{Validation of RNA-Seq Data Using qRT-PCR}

We collected four D. melanogaster larvae per treatment at $24 \mathrm{~h}$ post-infection with 100 symbiotic or axenic nematodes. Total RNA was extracted using the Invitrogen ${ }^{\mathrm{TM}}$ Ambion Trizol ${ }^{\mathrm{TM}}$ reagent following the manufacturer's instructions. RNA was eluted in $30 \mu \mathrm{L}$ nuclease-free water and concentration was measured using a NanoDrop (Thermo Scientific, Waltham, MA, USA). Complementary DNA (cDNA) was prepared using the iScript ${ }^{\mathrm{TM}}$ cDNA synthesis kit (Bio-Rad, Hercules, CA, USA) and $500 \mathrm{ng}$ of RNA sample in a total reaction volume of $20 \mu \mathrm{L}$. cDNA synthesis was carried out on a C1000 Thermal Cycler (Bio-Rad). PCR cycles included $25{ }^{\circ} \mathrm{C}$ for $5 \mathrm{~min}, 46^{\circ} \mathrm{C}$ for $20 \mathrm{~min}$, and $95^{\circ} \mathrm{C}$ for $1 \mathrm{~min}$. The resulting cDNA samples were diluted 1:10 in nuclease-free water and $1 \mu \mathrm{L}$ was used as template for qRT-PCR experiments using the iTaq ${ }^{\mathrm{TM}}$ Universal SYBR Green Supermix (Bio-Rad) on a CFX96 ${ }^{\mathrm{TM}}$ Real-Time System (Bio-Rad). The total reaction volume was $20 \mu \mathrm{L}$ and samples were run in technical duplicates for each set of primers (Table A2).

Table A1. Drosophila melanogaster strains used in this study.

\begin{tabular}{|c|c|c|c|}
\hline Gene & Mutant Genotype & Nature of Mutation & Background Strain \\
\hline PGRP-LE & $y^{1} w^{67 c 23}$ PGRP-LE $E^{112}$ & P-element activity & $y w$ \\
\hline Wengen & $\mathrm{P}\{\mathrm{GD} 3427\}_{\mathrm{V}} 9152$ & $\begin{array}{l}\text { Transposable element } \\
\text { insertion }\end{array}$ & $w^{1118}$ \\
\hline Domeless & NA & Homozygous viable & $w^{1118}$ \\
\hline Dawdle & $\begin{array}{l}\mathrm{w}^{*} ; \\
\mathrm{P}\{\text { GawB }\} \mathrm{daw}^{\mathrm{NP}} 4661 / \mathrm{CyO}\end{array}$ & P-element insertion & $w^{1118}$ \\
\hline Decapentaplegic & NA & Spontaneous & $w^{1118}$ \\
\hline
\end{tabular}

Table A2. Primer sequences used in quantitative RT-PCR assays.

\begin{tabular}{|c|c|c|c|c|}
\hline Gene & Accession Number & Primer & Sequence & $\operatorname{Tm}\left({ }^{\circ} \mathrm{C}\right)$ \\
\hline$P G R P-L E$ & CG8995 & $\begin{array}{l}\text { Forward } \\
\text { Reverse }\end{array}$ & $\begin{array}{l}\text { ATTGCAGAGTCCTCGGTTGTG } \\
\text { TTCACTGGTATTTTGGTCGGC }\end{array}$ & 61 \\
\hline MyD88 & CG2078 & $\begin{array}{l}\text { Forward } \\
\text { Reverse }\end{array}$ & $\begin{array}{l}\text { ATCTGGAACACTTCCTGGGC } \\
\text { CCACGAGAGCAGTCTGTCG }\end{array}$ & 61 \\
\hline Wengen & CG6531 & $\begin{array}{l}\text { Forward } \\
\text { Reverse }\end{array}$ & $\begin{array}{l}\text { ACCATCTGCGGTTCCATATACG } \\
\text { CTGCTCATACTCGGAGGACTT }\end{array}$ & 61 \\
\hline Domeless & CG14226 & $\begin{array}{l}\text { Forward } \\
\text { Reverse }\end{array}$ & $\begin{array}{l}\text { GGCGGCGACTTTAATCTGAG } \\
\text { GGTGTTGTTCAGGATTCGGAT }\end{array}$ & 61 \\
\hline Dawdle & CG16987 & $\begin{array}{l}\text { Forward } \\
\text { Reverse }\end{array}$ & $\begin{array}{l}\text { GGTGGATCAGCAGAAGGACT } \\
\text { CCCACTGATCCAGTGTTTGA }\end{array}$ & 61 \\
\hline Decapentaplegic & CG9885 & $\begin{array}{l}\text { Forward } \\
\text { Reverse }\end{array}$ & $\begin{array}{l}\text { CCTTGGAGCCTCTGTCGAT } \\
\text { TGCACTCTGATCTGGGATTTT }\end{array}$ & 61 \\
\hline RpL32 & CG7939 & $\begin{array}{l}\text { Forward } \\
\text { Reverse }\end{array}$ & $\begin{array}{l}\text { GATGACCATCCGCCCAGCA } \\
\text { CGGACCGACAGCTGCTTGGC }\end{array}$ & 61 \\
\hline
\end{tabular}

\section{References}

1. Poinar, G.O., Jr. The Natural History of Nematodes; Prentice Hall: Englewood Cliffs, NJ, USA, 1983.

2. Lacey, L.A.; Georgis, R. Entomopathogenic nematodes for control of insect pests above and below ground with comments on commercial production. J. Nematol. 2012, 44, 218-225. [PubMed]

3. Lacey, L.A.; Grzywacz, D.; Shapiro-Ilan, D.I.; Frutos, R.; Brownbridge, M.; Goettel, M.S. Insect pathogens as biological control agents: Back to the future. J. Invertebr. Pathol. 2015, 132, 1-41. [CrossRef] [PubMed]

4. Castillo, J.C.; Reynolds, S.E.; Eleftherianos, I. Insect immune responses to nematode parasites. Trends Parasitol. 2011, 27, 537-547. [CrossRef] [PubMed] 
5. Cooper, D.; Eleftherianos, I. Parasitic nematode immunomodulatory strategies: Advances and perspectives. Pathogens 2016, 14, 58. [CrossRef] [PubMed]

6. Peña, J.M.; Carrillo, M.A.; Hallem, E.A. Variation in the susceptibility of Drosophila to different entomopathogenic nematodes. Infect. Immun. 2015, 8, 1130-1138. [CrossRef] [PubMed]

7. Yadav, S.; Daugherty, S.; Shetty, A.C.; Eleftherianos, I. RNAseq analysis of the Drosophila response to the entomopathogenic nematode Steinernema. G3 (Bethesda) 2017, 7, 1955-1967. [CrossRef] [PubMed]

8. Martens, E.C.; Goodrich-Blair, H. The Steinernema carpocapsae intestinal vesicle contains a subcellular structure with which Xenorhabdus nematophila associates during colonization initiation. Cell Microbiol. 2005, 7, 1723-1735. [CrossRef] [PubMed]

9. Herbert, E.E.; Goodrich-Blair, H. Friend and foe: The two faces of Xenorhabdus nematophila. Nat. Rev. Microbiol. 2007, 5, 634-646. [CrossRef] [PubMed]

10. Goodrich-Blair, H. They've got a ticket to ride: Xenorhabdus nematophila-Steinernema carpocapsae symbiosis. Curr. Opin. Microbiol. 2007, 10, 225-230. [CrossRef] [PubMed]

11. Hao, Y.; Montiel, R.; Abubucker, S.; Mitreva, M.; Simões, N. Transcripts analysis of the entomopathogenic nematode Steinernema carpocapsae induced in vitro with insect hemolymph. Mol. Biochem. Parasitol. 2010, 169, 79-86. [CrossRef] [PubMed]

12. Richards, G.R.; Goodrich-Blair, H. Master of conquest and pillage: Xenorhabdus nematophila global regulators control transitions from virulence to nutrient acquisition. Cell Microbiol. 2009, 11, 1025-1033. [CrossRef] [PubMed]

13. McMullen, J.G.; Stock, S.P. In vivo and in vitro rearing of entomopathogenic nematodes (Steinernematidae and Heterorhabditidae). J. Vis. Exp. 2014, 22, 52096. [CrossRef]

14. Yadav, S.; Shokal, U.; Forst, S.; Eleftherianos, I. An improved method for generating axenic entomopathogenic nematodes. BMC Res. Notes 2015, 8, 461. [CrossRef] [PubMed]

15. Kounatidis, I.; Ligoxygakis, P. Drosophila as a model system to unravel the layers of innate immunity to infection. Open Biol. 2012, 2, 120075. [CrossRef] [PubMed]

16. Arefin, B.; Kucerova, L.; Dobes, P.; Markus, R.; Strnad, H.; Wang, Z.; Hyrsl, P.; Zurovec, M.; Theopold, U. Genome-wide transcriptional analysis of Drosophila larvae infected by entomopathogenic nematodes shows involvement of complement, recognition and extracellular matrix proteins. J. Innate Immun. 2014, 6, 192-204. [CrossRef] [PubMed]

17. Castillo, J.C.; Creasy, T.; Kumari, P.; Shetty, A.; Shokal, U.; Tallon, L.J.; Eleftherianos, I. Drosophila anti-nematode and antibacterial immune regulators revealed by RNA-seq. BMC Genom. 2015, 11, 519. [CrossRef] [PubMed]

18. Binda-Rossetti, S.; Mastore, M.; Protasoni, M.; Brivio, M.F. Effects of an entomopathogen nematode on the immune response of the insect pest red palm weevil: Focus on the antimicrobial response. J. Invertebr. Pathol. 2016, 133, 110-119. [CrossRef] [PubMed]

19. Tauszig-Delmasure, S.; Bilak, H.; Capovilla, M.; Hoffmann, J.A.; Imler, J.L. Drosophila MyD88 is required for the response to fungal and Gram-positive bacterial infections. Nat. Immunol. 2002, 3, 91-97. [CrossRef] [PubMed]

20. Yang, H.; Kronhamn, J.; Ekström, J.O.; Korkut, G.G.; Hultmark, D. JAK/STAT signaling in Drosophila muscles controls the cellular immune response against parasitoid infection. EMBO Rep. 2015, 16, 1664-1672. [CrossRef] [PubMed]

21. Gramates, S.L.; Marygold, S.J.; dos Santos, G.; Urbano, J.; Antonazzo, G.; Matthews, B.B.; Rey, A.J.; Tabone, C.J.; Crosby, M.A.; Ennert, D.B.; et al. FlyBase at 25: Looking into the future. Nucleic Acid Res. 2017, 45, D663-D671. [CrossRef] [PubMed]

22. Ekengren, S.; Tryselius, Y.; Dushay, M.S.; Liu, G.; Steiner, H.; Hultmark, D. A humoral stress response in Drosoph. Curr. Biol. 2001, 11, 714-718. [CrossRef]

23. White, G.F. A method for obtaining infective nematode larvae from cultures. Science 1927, 66, $302-303$. [CrossRef] [PubMed]

24. Hughes, T.R. 'Validation' in genome-scale research. J. Biol. 2009, 8, 3. [CrossRef] [PubMed]

25. Castillo, J.C.; Shokal, U.; Eleftherianos, I. Immune gene transcription in Drosophila adult flies infected by entomopathogenic nematodes and their mutualistic bacteria. J. Insect Physiol. 2013, 59, 179-185. [CrossRef] [PubMed] 
26. Hwang, J.; Park, Y.; Kim, Y.; Hwang, J.; Lee, D. An entomopathogenic bacterium, Xenorhabdus nematophila, suppresses expression of antimicrobial peptides controlled by Toll and Imd pathways by blocking eicosanoid biosynthesis. Arch. Insect Biochem. Physiol. 2013, 83, 151-169. [CrossRef] [PubMed]

27. Hussa, E.A.; Casanova-Torres, A.M.; Goodrich-Blair, H. The global transcription factor Lrp controls virulence modulation in Xenorhabdus nematophila. J. Bacteriol. 2015, 197, 3015-3025. [CrossRef] [PubMed]

28. Aymeric, J.L.; Givaudan, A.; Duvic, B. Imd pathway is involved in the interaction of Drosophila melanogaster with the entomopathogenic bacteria, Xenorhabdus nematophila and Photorhabdus luminescens. Mol. Immunol. 2012, 47, 2342-2348. [CrossRef] [PubMed]

29. Hallem, E.A.; Rengarajan, M.; Ciche, T.A.; Sternberg, P.W. Nematodes, bacteria, and flies: A tripartite model for nematode parasitism. Curr. Biol. 2007, 17, 898-908. [CrossRef] [PubMed]

30. Eleftherianos, I.; Castillo, J.C.; Patrnogic, J. TGF- $\beta$ signaling regulates resistance to parasitic nematode infection in Drosophila melanogaster. Immunobiology 2016, 221, 1362-1368. [CrossRef] [PubMed]

31. Noselli, S.; Angès, F. Roles of the JNK signaling pathway in Drosophila morhogenesis. Curr. Opin. Genet. Dev. 1999, 9, 466-472. [CrossRef]

32. Stronach, B.E.; Perrimon, N. Stress signaling in Drosophila. Oncogene 1999, 18, 6172-6182. [CrossRef] [PubMed]

33. Rämet, M.; Lanot, R.; Zachary, D.; Manfruelli, P. JNK signaling pathway is required for efficient wound healing in Drosophila. Dev. Biol. 2002, 241, 145-156. [CrossRef] [PubMed]

(C) 2018 by the authors. Licensee MDPI, Basel, Switzerland. This article is an open access article distributed under the terms and conditions of the Creative Commons Attribution (CC BY) license (http://creativecommons.org/licenses/by/4.0/). 